Conclusion STX18-AS1 is the first long noncoding RNA influencing CHD risk identified from GWAS. The mechanism involves downregulation of the NKX2-5 gene through epigenetic mechanisms.

Conflict of interest No

\section{BS3 NOTCH LIGAND JAG1 PROMOTES ENDOTHELIAL-TO- MESENCHYMAL TRANSITION AND ATHEROSCLEROSIS AT REGIONS OF DISTURBED FLOW}

Celine Souilhol ${ }^{*}$, Lindsay Canham, Jiu Xueqi, Paul Evans. University of Sheffield

\subsection{6/heartjnl-2019-BCS.167}

Introduction Atherosclerosis is a chronic inflammatory disease marked by hardening and thickening of the arteries. The disease develops predominantly at arterial branches and bends. These atheroprone areas are subjected to disturbed blood flow, which generates low and oscillatory wall shear stress (LOSS), a frictional force exerted on endothelial cells (EC). LOSS increases EC inflammatory activation and drives endothelial-to-mesenchymal transition (EndoMT), which promotes atherosclerosis. The molecular basis of EC responses to shear stress is not fully elucidated.

Notch signaling is a major regulator of vascular development and homeostasis. It plays a critical role in communication between EC, initiated by the interaction of a receptor (i. e. Notch1 or 4) with a ligand (Dll4 or Jag1) present on a neighbouring cell. A recent study revealed that Notch1 is a mechanosensor protecting adult arteries from developing atherosclerosis (Mack et al., 2017, Nat Commun.;8(1):1620). However the role of other Notch actors in this disease is unknown. Here we investigated the role of the Notch ligand Jag1 in EC pathophysiology and atherosclerosis.

Methods To study the effect of shear stress on Notch actors, Human Coronary Aortic EC (HCAEC) were exposed to LOSS (4dynes $/ \mathrm{cm}^{2} \pm 1 \mathrm{~Hz}$ ) versus high shear stress (12dynes/ $\mathrm{cm}^{2}$ ) using a parallel plate system (Ibidi). After 2 to 4 days of culture under flow, expression levels of transcripts and proteins were assessed by qRT-PCR and western blotting. The function of Jag1 in HCAEC exposed to LOSS was studied using neutralizing antibodies. The function of Jag1 in EC was studied in vivo by tamoxifen inducible conditional deletion in mice (CDH5Cre/Ert2; Jag1fl/fl). The EC phenotype was analysed by immunostaining, qRT-PCR and western blotting.

Results qRT-PCR and en face staining revealed that Jag1 is enriched at LOSS sites in both the pig $(p<0.05)$ and mouse aorta $(p<0.05)$. We concluded that LOSS is responsible for focal Jag1 expression at atheroprone areas because it induced Jag1 in HCAEC $(\mathrm{p}<0.05)$. We then investigated whether Jag1 influences EC dysfunction in response to LOSS. Blocking Jag1 activity reduced expression of inflammatory markers (e.g. VCAM-1, E-Selectin and MCP-1) as well as mesenchymal markers (e.g. Snail, N-Cadherin and SMA) in HCAEC exposed to LOSS. It also significantly increased proliferation. Thus LOSS activation of Jag1 drives EC activation and EndoMT and reduces proliferative potential. Co-staining of Jag1 with markers of inflammation and
EndoMT revealed a strong colocalization in the mouse aorta. Jag1 was also detected in EC above atherosclerotic plaques. To investigate whether endothelial Jag1 plays a role in atherosclerosis, we carried out an inducible knockout of Jag1 in EC, which resulted in a decrease of plaque formation.

Conclusion Our data reveal a critical role for the Notch ligand Jag1 as a pro-atherogenic factor, controlling inflammation, EC fate and proliferation at atheroprone sites.

Conflict of interest none

\section{BS4 IRON DEFICIENCY IN PULMONARY ARTERIAL SMOOTH MUSCLE CELLS INDUCES PULMONARY ARTERIAL HYPERTENSION THROUGH ENDOTHELIN-1}

${ }^{1}$ Samira Lakhal-Littleton*, ${ }^{2}$ Alexi Crosby, ${ }^{1}$ Goran Mohammad, ${ }^{1}$ Matthew Frise, ${ }^{1}$ Carolyn Carr, ${ }^{1}$ Paul Loick, ${ }^{1}$ Peter Robbins. ' University of Oxford; ${ }^{2}$ University of Cambridge

\subsection{6/heartjnl-2019-BCS.168}

Introduction Iron deficiency augments hypoxic pulmonary arterial pressure in healthy individuals and exacerbates pulmonary arterial hypertension (PAH) in patients, even in the absence of anaemia. In supplementation has been shown to be beneficial in both settings. The mechanisms underlying the detrimental effects of iron deficiency and the beneficial effects of iron supplementation are not known, owing to a lack of understanding of how specific cells of the pulmonary vasculature respond to changes in iron levels. The iron export protein ferroportin (FPN) and its antagonist peptide hepcidin control systemic iron levels by regulating its release from the gut, spleen and liver, the sites of iron absorption, recycling and storage, respectively. We found FPN to be present in pulmonary arterial smooth muscle cells (PASMCs) and to be regulated by hepcidin. Therefore, we set out to interrogate the physiological function of the hepcidin/FPN axis in PASMCs.

Methods We generated a murine model with a smooth muscle-specific knock-in of fpn C326Y, which encodes a FPN with intact iron export function but impaired hepcidin binding. We then studied pulmonary hemodynamics and cardiac function over time.

Results While retaining normal systemic iron and haemoglobin levels, this model developed $\mathrm{PAH}$ and right heart failure as a consequence of intracellular iron deficiency and increased expression of the vasoconstrictor endothelin-1 (ET-1) specifically within PASMCs. PAH was prevented and reversed by intravenous iron treatment and by the ET receptor antagonist BQ-123. The regulation of ET-1 by iron was further demonstrated in healthy humans exposed to hypoxia and in PASMCs from $\mathrm{PAH}$ patients.

Conclusion This study presents the first evidence that intracellular iron deficiency localised specifically within PASMCs is sufficient to impair pulmonary vascular function, even in the absence of anaemia (fig 1). It offers a mechanistic underpinning for the known effects of iron availability on the pulmonary vasculature in the human setting.

Conflict of interest None 